\title{
Prolactin Levels in Umbilical Cord Serum and Its Relation to Fetal Adrenal Activity in Newborns of Women with Pregnancy-Induced Hypertension
}

\author{
C. R. PARKER, JR., G. D. V. HANKINS, B. R. CARR, N. F. GANT, P. C. MACDONALD, AND \\ J. C. PORTER \\ Cecil H. and Ida Green Center for Reproductive Biology Sciences and the Departments of Obstetrics and \\ Gynecology, Biochemistry, and Physiology, The University of Texas Southwestern Medical School,
} Dallas, Texas 75235

\begin{abstract}
The effect of hypertension in pregnant women on fetal maturation is an issue of considerable importance. Because of a possible role of prolactin in fetal adrenal steroidogenesis and in fetal lung maturation, we have investigated the relationship between hypertension in pregnant women and levels of prolactin and dehydroepiandrosterone sulfate in serum of newborn infants. It was found that with the mild-to-moderate form of pregnancyinduced hypertension (PIH), there was little effect on prolactin levels in newborn serum. In newborns of women with severe PIH, however, serum prolactin levels were significantly greater $(p<0.01)$ than those in newborns of women with uncomplicated pregnancies. Conversely, umbilical serum concentrations of dehydroepiandrosterone sulfate in newborns of women with severe PIH were significantly less $(p<0.05)$ than those in newborns of women with uncomplicated pregnancies. These findings are supportive of the view that pituitary function and adrenocortical function of fetuses of women with PIH are different from those of fetuses of normotensive women. These findings are suggestive that PIH alters the function of the fetal pituitary and adrenal cortex. (Pediatr Res 20: 876-878, 1986)
\end{abstract}

\section{Abbreviations}

PIH, pregnancy-induced hypertension

DS, dehydroepiandrosterone sulfate

The effects of disorders of pregnant women on the processes of human fetal development are varied. Perhaps the most common complication of pregnancy is PIH. In the current study, we investigated the effects of PIH on the levels of prolactin and DS in serum of newborns. Prolactin was chosen for study since it is a major secretory product of the fetal pituitary that has been proposed to serve a role in fetal adrenal steroidogenesis (1-6), and also in fetal lung maturation (7-10). DS was chosen for study since it is the major secretory product of the fetal adrenal cortex and is the principal precursor of estrogens formed in the placenta during human pregnancy (11).

Received February 20,1986; accepted April 28, 1986.

Correspondence and reprint requests to Dr. C. Richard Parker, Jr., Department of Obstetrics and Gynecology, University of Alabama at Birmingham, University Station, Birmingham, AL 35294.

These studies were supported, in part, by USPHS Grants HD14513 and HDI3912.

\section{METHODS}

The subjects of this study were 157 newborn infants of women who were normotensive and whose pregnancies were considered to be otherwise uncomplicated and 71 newborn infants of women whose pregnancies were complicated by PIH. All newborns were delivered between 28 and 40 wk of gestation: gestational age being the number of weeks since the last menstrual period minus 2 wk. Newborns of hypertensive women with additional complications (i.e. chronic hypertension, diabetes, etc.) were omitted from this study. PIH was classified as severe when the onset of hypertension occurred prior to or during the $32 \mathrm{nd}$ wk of gestation. In instances when the onset of hypertension occurred after the 32nd wk of gestation, PIH was classified according to the magnitude of diastolic blood pressure elevation. Women having a persistent diastolic blood pressure of more than $110 \mathrm{~mm} \mathrm{Hg}$ were classified as having severe PIH. Women having a persistent diastolic blood of $100-110 \mathrm{~mm} \mathrm{Hg}$ were classified as having mild-to-moderate PIH. Of those women having a diastolic blood pressure of less than $100 \mathrm{~mm} \mathrm{Hg}$, those having an increase of 20 $\mathrm{mm} \mathrm{Hg}$ in diastolic blood pressure over the level at midpregnancy also were considered to have mild-to-moderate PIH. There were no differences in the anesthetic and analgesic agents used during delivery for normotensive as opposed to the hypertensive subjects of this study. Most of the women having PIH were administered $\mathrm{MgSO}_{4}$ near the time of delivery; some of those having severe $\mathrm{PIH}$ (diastolic blood pressure $>110 \mathrm{~mm} \mathrm{Hg}$ ) also were treated with hydralazine during the peripartal period. None of the hypertensive women was administered phenothiazines, which are known to increase prolactin secretion, during pregnancy. Neither $\mathrm{MgSO}_{4}$ nor hydralazine are known to influence adrenal steroid secretion or pituitary prolactin secretion.

At the time of delivery, the umbilical cord was separated from the infant and blood (mixed arterial and venous) was drained from a segment of the umbilical cord. After clot formation, the serum was separated by centrifugation and stored at $-20^{\circ} \mathrm{C}$ until assayed. Serum prolactin was measured by radioimmunoassay procedures $(12,13)$ by use of antiserum and reference standards provided by the National Institute of Health. DS was quantified by a radioimmunoassay procedure (14). Some of the subjects of this study were part of earlier studies of a different context and the results are reported elsewhere (15). Since we previously found that method of delivery was not a factor that affected newborn serum levels of prolactin (7) or DS (15), data for newborns were pooled for statistical evaluation without regard to whether delivery occurred by vaginal delivery or cesarean section, with or without labor. The data were subjected to unpaired $t$ test, analysis of variance. Neuman-Keuls multiple range test, and linear regression analysis (16). These studies were subjected to prior review 
and were approved by the Institutional Review Board of the University of Texas Health Science Center at Dallas.

\section{RESULTS}

The concentrations of prolactin in umbilical cord serum of 71 newborns by hypertensive women were compared with those in 157 newborns of women having uncomplicated pregnancies (Fig. 1). Significant variation in prolactin levels among the groups of newborns was found to exist at $32-35 \mathrm{wk}(\mathrm{F}=8.18, p<0.001)$ and at $36-40 \mathrm{wk}(\mathrm{F}=4.93, p<0.01)$. Serum prolactin levels in newborns of women with no pregnancy complications and of women with mild-to-moderate PIH were similar from 32-40 wk gestation. On the other hand, serum prolactin levels in newborns of women with severe PIH were significantly higher at 28-31 ( $p$ $<0.001), 32-35(p<0.001)$, and 36-40 wk of gestation $(p<$ 0.01 ) than those in newborns of women whose pregnancy was uncomplicated and also were higher $(p<0.025)$ than those in newborns delivered at term of women having mild-to-moderate PIH.

We also examined the relationship between concentrations in umbilical cord serum of DS and prolactin that had been determined in the same set of newborns $(n=164)$. Significant variation in umbilical serum levels of $\mathrm{DS}(\mathrm{F}=5.74, p=0.005)$ and prolactin $(\mathrm{F}=5.83, p=0.004)$ as a function of gestational age occurred only in newborns of women having uncomplicated pregnancies (Fig. 2). Umbilical serum levels of DS and prolactin in newborns of women having uncomplicated pregnancies were significantly higher at $36-40 \mathrm{wk}$ of gestation than at $28-31 \mathrm{wk}$ of gestation $(p<0.01$ and $<0.01$, respectively). The gestational age-related increases in umbilical serum levels of DS and prolactin in newborns of hypertensive women were not statistically significant. Umbilical serum levels of DS in newborns of women having severe PIH were significantly lower than those in women having uncomplicated pregnancies at 32-35 wk of gestation ( $p$ $<0.05)$ and at $36-40 \mathrm{wk}$ of gestation $(p<0.05)$.

The data presented in Figure 2 are suggestive that DS and prolactin concentrations in umbilical cord serum rise concomitantly during the latter third of fetal development. Indeed, a near significant, positive correlation between DS and prolactin levels (Pearson's $\mathrm{R}=0.3958, p=0.055$ ) was found among newborns of normotensive women at 28-31 wk of gestation. There was, however, no apparent correlation between DS and prolactin among newborns of normotensive or hypertensive women at
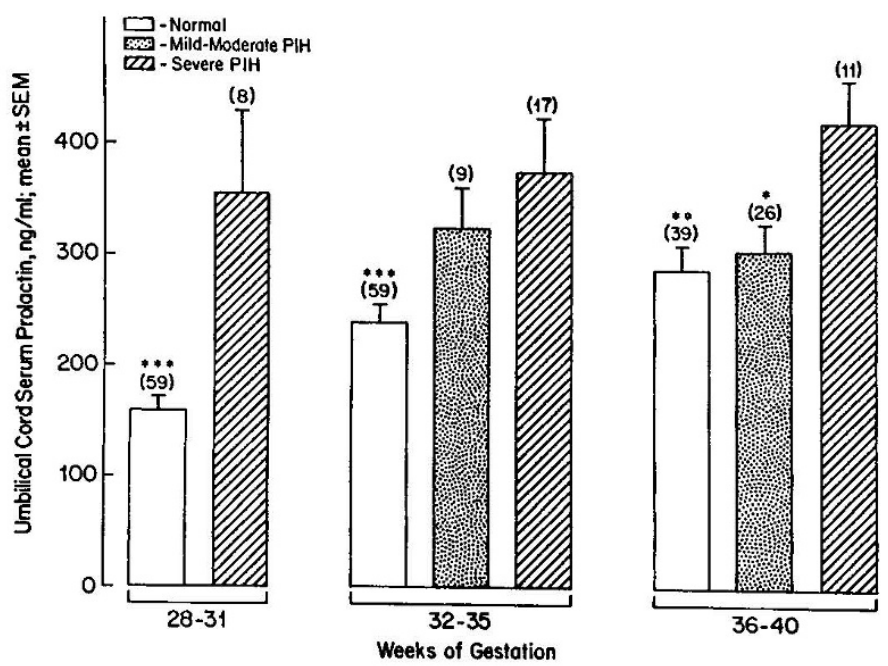

Fig. 1. Prolactin concentrations in umbilical cord serum of newborn infants of women whose pregnancies were normal or else were complicated by PIH. The data are presented as the means \pm SEM. The number of newborns in each group is indicated at the top of each bar. ${ }^{*} p<0.025$, ${ }^{* *} p<0.01$, and ${ }^{* * *} p<0.001$ compared with values from serum of newborns of women having severe PIH.

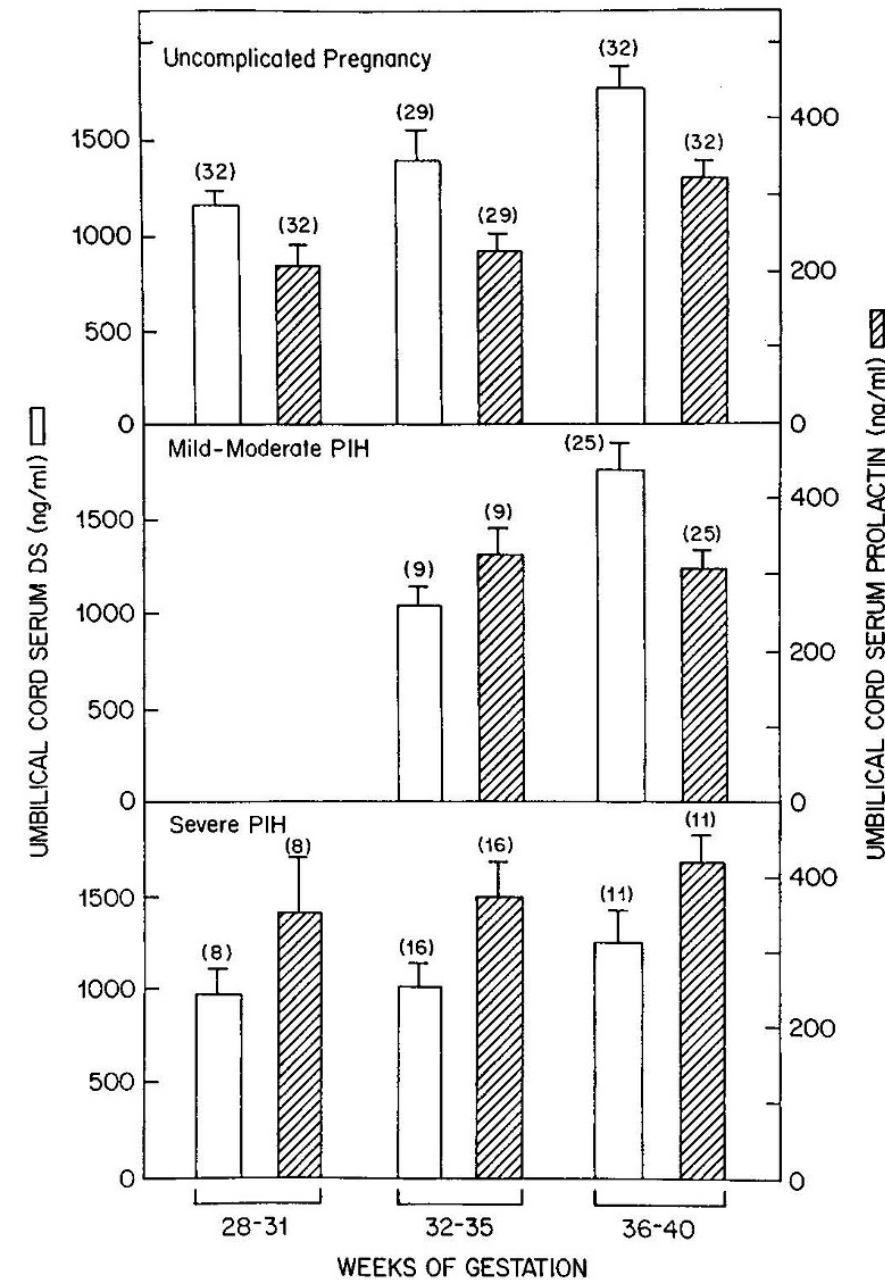

Fig. 2. Umbilical cord serum concentrations of prolactin and DS in newborn infants of women whose pregnancies were normal or else were complicated by PIH. The data are subdivided according to gestational age of the newborn and are presented as the means \pm SEM. The number of newborns in each group is indicated at the top of each bar.

other gestational ages. When concentrations in umbilical cord serum of DS and prolactin were subjected to linear regression analysis for all newborns within a given age range, we again found that there was no apparent correlation between umbilical serum levels of DS and prolactin amongst newborns delivered at $28-31(\mathrm{R}=0.0089), 32-35(\mathrm{R}=-0.056)$, or $36-40 \mathrm{wk}$ of gestation $(\mathrm{R}=-0.204, p=0.09)$.

The birth weights of newborns of women having PIH were lower than those of newborns of normal women at 28-31 wk of gestation $(1473 \pm 58$ and $1707 \pm 48 \mathrm{~g}$, respectively; $p<0.01)$ and at 32-35 wk of gestation $(2092 \pm 63$ and $2256 \pm 41 \mathrm{~g}$, respectively; $p<0.05$ ). At term, however, the birth weights of both groups were similar. When the data for newborns of normal women and for newborns of women having PIH were grouped separately according to sex, we found that birth weights, 5-min Apgar scores, and umbilical cord serum concentrations of DS and of prolactin in male infants were similar to those in female infants at each gestational period. Lastly, we also sought to determine whether intrauterine distress was related to any of the variables measured in this study. When the data for all newborns were grouped according to 5-min Apgar scores, newborns showing signs of intrauterine distress $(5$-min Apgar $<7$ ) were found to have a lower birth weight than newborns having a normal 5min Apgar $(\geq 7)$ at $28-31 \mathrm{wk}(1427 \pm 85$ versus $1705 \pm 42 \mathrm{~g}$, mean $\pm \mathrm{SE} ; p<0.01)$ and at $36-40 \mathrm{wk}$ of gestation $(2668 \pm 174$ versus $3079 \pm 56 \mathrm{~g}, p<0.05)$. Umbilical cord serum levels of 
prolactin and DS in newborns having 5-min Apgar scores of $<$ 7 were, however, similar to those having normal 5-min Apgar scores at each gestational period.

\section{DISCUSSION}

Although the effects of many maternal pregnancy complications on gross parameters of neonatal outcome, e.g. size and weight, are well documented, the effects of such pregnancy complications on endocrine maturation of the fetus are largely undocumented. The results of prior studies are suggestive that PIH is associated with impaired function of the fetal zone of the fetal adrenal but normal development of the neocortical zone of the fetal adrenal $(15,17-20)$. Our current data are supportive of the view that PIH leads to maldevelopment of the fetal zone of the adrenal, and thus to decreased DS production by the fetal adrenals.

Since prolactin has been implicated as an agent that acts to stimulate secretion of adrenal $\mathrm{C}_{19}$-steroids such as DS but not $\mathrm{C}_{21}$-steroids such as cortisol (1-6), we considered the possibility that PIH might lead to inhibition of fetal adrenal DS secretion by means of decreasing fetal pituitary prolactin secretion. Such was not the case. In fact, in newborns that had the greatest impairment in fetal adrenal DS secretion, viz., fetuses of women with severe $\mathrm{PIH}$, the levels of serum prolactin were significantly greater than those in newborns of women having no pregnancy complications. Grosso et al. (10) also found that newborns of hypertensive women tended to have higher serum levels of prolactin than those in newborns of women having uncomplicated pregnancies. The fact that newborns of women with severe hypertension have higher than normal concentrations of serum prolactin may be salutatory since such newborns commonly do not develop respiratory distress syndrome when delivered prematurely (21). On the other hand, prematurely delivered newborns that develop respiratory distress syndrome often have lower serum levels of prolactin than do similarly aged newborns whose lung function is normal (7-10).

It is known that fetal pituitary secretion of prolactin increases progressively during normal human gestation $(22,23)$. This is thought to be due, in part, to the ever increasing production of estrogen that is characteristic of human pregnancy. The mechanism(s) responsible for higher than normal rates of prolactin secretion in newborns of hypertensive women is, however, unknown. The subjects of this study, although carefully selected, represent a heterogeneous group for which it is virtually impossible to correct or account for the numerous variables that may or may not influence fetal adrenal and/or pituitary function. We believe, however, that the observed differences between newborns of normotensive women and of hypertensive women likely are not attributable to the effects of anesthetic and analgesic agents used during delivery, method of delivery, or sex-ratio differences of the newborn populations.

In women with severe PIH, there is known to be decreased uteroplacental blood flow and decreased clearance of placental estrogens into the maternal circulation (24). Conversely, when removal of steroids into the maternal circulation is impaired, the opportunity for passage of placentally formed steroids into the fetal compartment likely is increased with PIH. Thus, it is possible [and preliminary evidence has been presented in support of this possibility (25)] that hyperestrogenism may paradoxically exist in some fetuses that produce lower than normal amounts of estrogen precursor, i.e. DS, and whose mothers are hypoestrogenic. Such an occurrence could be expected to lead to increased fetal pituitary prolactin secretion (26). Alternatively, increased secretion of prolactin in newborns believed to be "stressed" in utero as a result of maternal hypertension might arise by way of a mechanism(s) similar to that which leads to stress-induced increase in prolactin secretion in adults. Among the entire population of newborns, however, we found no relationship between 5-min Apgar scores and umbilical cord serum levels of prolactin or DS. It also must be recognized that "stress", per se, in the fetus in particular is difficult to define, and concepts formulated for "stress" responses in adults may not be applicable to the human fetus.

\section{REFERENCES}

1. Carter JN, Tyson JE, Warne GL, McNeilly AS, Faiman C, Friesen HG 1977 Adrenocortical function in hyperprolactinemic women. J Clin Endocrinol Metab 45:973-979

2. Vermeulen A, Suy E, Rubens R 1977 Effect of prolactin on plasma DHEA(S) levels. J Clin Endocrinol Metab 44:1222-1225

3. Brown TJ, Glinz B, Milne CM, Oakey RE 1981 Stimulation by polypeptides of dehydroepiandrosterone sulfate synthesis in human foetal adrenal slices. J Endocrinol 9:111-122

4. Higuchi K, Nawata H, Maki J, Higashizima M, Kato K-I, Ibayashi H 1984 Prolactin has a direct effect on adrenal androgen secretion. J Clin Endocrinol Metab 59:714-718

5. Pepe GJ, Albrecht ED 1985 Regulation of baboon fetal adrenal androgen production by adrenocorticotropic hormone, prolactin and growth hormone. Biol Reprod 33:545-550

6. Schiebinger RJ, Chrousos GP, Cutler GB Jr, Loriaux DL 1986. The effect of serum prolactin on plasma adrenal androgens and the production and metabolic clearance rate of dehydroepiandrosterone sulfate in normal and hyperprolactinemic subjects. J Clin Endocrinol Metab 62:202-209

7. Hauth JC, Parker CR Jr, MacDonald PC, Porter JC, Johnston JM 1978 A role of fetal prolactin in lung maturation. Obstet Gynecol 51:81-88

8. Gluckman PD, Ballard PL, Kaplan SL, Liggins GC, Grumbach MM 1978 Prolactin in umbilical cord blood and the respiratory distress syndrome. $\mathbf{J}$ Pediatrics 93:1011-1014

9. Smith YF, Mullon DK, Hamosh M, Scanlon JW, Hamosh P 1979 Serum prolactin and respiratory distress syndrome in the newborn. Pediatr Res 14:93-95

10. Grosso DS, MacDonald CP, Thomasson JE, Christian CD 1980 Relationship of newborn serum prolactin levels to the respiratory distress syndrome and maternal hypertension. Am J Obstet Gynecol 137:569-574

11. Siiteri PK, MacDonald PC 1966 Placental estrogen biosynthesis during human pregnancy. J Clin Endocrinol Metab 26:751-761

12. Hwang P, Guyda H, Friesen H 1971 A radioimmunoassay for human prolactin. Proc Natl Acad Sci USA 68:1902-1906

13. Sinha YN, Selby FW, Lewis UR, VanderLaan WP 1973 A homologous radioimmunoassay for human prolactin. J Clin Endocrinol Metab 36:509516

14. Buster J, Abraham GE 1977 Radioimmunoassay of dehydroepiandrosterone sulfate. Anal Letters 5:543-551

15. Parker CR Jr, Hankins GDV, Carr BR, Leveno KJ, Gant NF, MacDonald PC 1984 The effect of hypertension in pregnant women on fetal adrenal function and fetal plasma lipoprotein-cholesterol metabolism. Am J Obstet Gynecol 150:263-269

16. Zar JH 1974 Biostatical Analysis. Prentice-Hall, Inc, Englewood Cliffs, NJ.

17. Talbert LM, Easterling WE 1967 Factors influencing urinary estrogen excretion in pregnancy. Am J Obstet Gynecol 99:923-929

18. Cleary RE, Pion R 1969 Relationship of $\mathrm{C}_{19}$-steroid excretion in the newborn to maternal urinary estriol. Am J Obstet Gynecol 104:166-171

19. Reynolds JW, Turnipseed MR, Mirkin BL 1975 Adrenal cortical function in abnormal newborn infants. J Steroid Biochem 6:669-672

20. Hardy MJ, Humeida AK, Bahijri SM, Basalamah AH 1981 Late third trimester unconjugated serum oestriol levels in normal and hypertensive pregnancy relation to birth weight. Br J Obstet Gynecol 88:976-982

21. Farrell PM, Avery ME 1975 Hyaline membrane disease. Am Rev Respir Dis 111:657-688

22. Winters AJ, Colston C, MacDonald PC, Porter JC 1975 Fetal plasma prolactin levels. J Clin Endocrinol Metab 41:626-629

23. Aubert ML, Grumbach MM, Kaplan SL 1975 The ontogenesis of human fetal hormones III Prolactin. J Clin Invest 56:155-164

24. Worley RJ, Everett RB, Madden JD, MacDonald PC, Gant NF 1978 Fetal considerations. Metabolic clearance rate of maternal plasma dehydroisoandrosterone sulfate. Semin Perinatol 2:15-28

25. Hankins G, Carr BR, Parker CR Jr 1983 Placental processing of estrogen precursors in normal and complicated pregnancies. Proceedings of the 30th annual meeting of the Society for Gynecologic Investigation, March 17-20, Washington DC (abstr 332)

26. Yen SSC, Ehara Y, Siler TM 1974 Augmentation of prolactin secretion by estrogen in hypogonadal women. J Clin Invest 53:652-655 